\section{IDDF2019-ABS-0046 ENDOSCOPIC PRACTICE OF VARICEAL ERADICATION IN CHINESE LIVER CENTER}

${ }^{1}$ Jinni Luo*, 'Xing Wang, 'Shaoyan Guo, ${ }^{2}$ Hongli Shang, 'Wurina Bai, 'Junyu Liu, ${ }^{1}$ Xiaoying Wu, ${ }^{1}$ Bin Wu. ${ }^{1}$ Department of Gastroenterology, The Third Affiliated Hospital of Sun Yat-sen University, Guangzhou, China; ${ }^{2}$ Department of Nephrology, The Third Affiliated Hospital of Sun Yat-sen University, Guangzhou, China

\subsection{6/gutjnl-2019-IDDFabstracts.248}

Background Esophagogastric varices (EGV) is a major consequence of portal hypertension highly associated with dreadful variceal hemorrhage, and complete variceal eradication may greatly improve recurrence and prognosis. Little is known about the endoscopic resources invested for EGV eradication in China. This observational study aimed to clarify the practice of variceal eradication by modern combination endoscopic strategies and investigate factors associated with treatment sessions.

Methods Clinical data were extracted from the endoscopic recording database and medical charts. Cirrhotic patients underwent the initial endoscopic treatment in our department and followed up for more than 2 months were included between Oct 2011 to Jun 2018. Endoscopic sequential therapy (EST) is an integrated protocol comprising endoscopic cyanoacrylate injection (ECI) sequenced by repeated sessions of endoscopic band ligation (EBL) and/or endoscopic injection sclerotherapy (EIS), and this protocol was performed for every patient. Patients were intensively followed up until complete EGV eradication.

Results Among the 337 patients recruited to follow up, 291 $(86.4 \%)$ who successfully reached variceal eradication were included for analysis. A total of 1029 episodes of endoscopic treatments were performed for the patients. Among them, 132 (45.4\%) patients had Child-Pugh class B or C liver disease, and $46(15.8 \%)$ had MELD score equal or over 15. EBL was the most prevalent method used in 283 (97\%) patients, followed by ECI for 121 patients (41.6\%) and EIS for 53 patients (18.2\%). A mean 3.5 sessions of endoscopic therapy was needed to reach complete variceal eradication during an average of 9.6 months (range, 24 days-75 months). The median endoscopic interval between sessions for each patient was 2.0 months (IQR 1.3-3.0). There was a significant positive association between portal vein diameter and both the number of endoscopic sessions $(\mathrm{P}<0.001$, Spearman correlation) and time duration $(\mathrm{P}=0.049$, Spearman correlation) needed to obtain variceal eradication.

Conclusions Most cirrhotic patients with EGV undergoing EST would achieve variceal eradication. EVL is the mainstay of the endoscopic method. Portal vein diameter is related to endoscopic resources invested for variceal eradication. Interval between sessions is far from optimal in China and physicians should pay more attention to patient compliance in clinical practice.

\section{IDDF2019-ABS-0051 A COMPARISON OF RISK SCORE MODELS TO PREDICT THE RISK OF HCC IN CHRONIC HEPATITIS B PATIENTS IN THAILAND}

\footnotetext{
${ }^{1}$ Thanachote Kamalapirat*, ${ }^{1}$ Teerapat Ungtrakul, ${ }^{2}$ Kamonwan Soonklang, ${ }^{2}$ Jiraporn Dechma, ${ }^{2}$ Pitchayachuda Chunnuan, ${ }^{2}$ Pattama Kusuman, ${ }^{2}$ Charinthip Pothijaroen, ${ }^{2}$ Kesinee Yingcharoen. ${ }^{1}$ Faculty of Medicine and Public Health, Princess Chulabhorn College of Medical Sciences, Chulabhorn Royal Academy, Thailand; ${ }^{2}$ Chulabhorn Hospital, Princess Chulabhorn College of Medical Sciences, Chulabhorn Royal Academy, Thailand
}

Background Chronic hepatitis $\mathrm{B}$, a major risk factor for cirrhosis and hepatocellular carcinoma(HCC), is endemic in Thailand. Currently, there is no tool for predicting the accurate risk of HCC in chronic hepatitis B patients. The aim of this study was to compare among previously developed risk score models and identify the most accurate risk score to predict the risk of HCC in chronic hepatitis B patients in Thailand.

Methods A retrospective cohort study including 2,208 chronic hepatitis B patients from the Screening for Hepatocellular Carcinoma Program at Chulabhorn hospital during July 2010-September 2017. The baseline characteristics, laboratory and radiologic data of chronic hepatitis $\mathrm{B}$ patients during their initial visit were obtained and calculated for the GAG-HCC, CU-HCC and REACH-B scores. We performed receiver operating curve(ROC) for each score and compared the AUROC using chi-squared test.

Results Of 2,208 chronic hepatitis B patients, one-fifth was HBeAg positive $(377,17.1 \%)$ and almost all the populations was non-cirrhotic(2,148, 97.1\%). Nearly twenty percent of our populations was prescribed nucleos(t)ides analogues $(434$, 19.7\%). After 6 years follow-up, 20 patients(9.05\%) developed HCC. GAG-HCC, CU-HCC and REACH-B scores identified 44(1.99\%), 65(2.94\%) and 800(36.23\%) high-risk patients based on cut-off values at the initial visit. The sensitivity of GAG-HCC, CU-HCC and REACH-B scores were $25 \%, 35 \%$ and $85 \%$, respectively. The specificity of GAGHCC, CU-HCC and REACH-B scores were 98.22\%, 97.35\% and 64.21\%, respectively. The AUROC for GAGHCC is $0.80(95 \%$ CI, $0.69-0.91)$, CU-HCC is $0.73(95 \%$ CI, $0.60-0.85)$ and REACH-B is $0.79(95 \%$ CI, 0.67-0.91). Chi-squared test performed between the three AUROCs showed no difference between the three scores $(p=0.154)$. (figure 1)

Conclusions There is no significant difference between the three risk score models in predicting the risk of developing hepatocellular carcinoma in Thai population. Additional variables such as anti-viral medications could be considered to develop an improved score model more relevant to the Thai population.

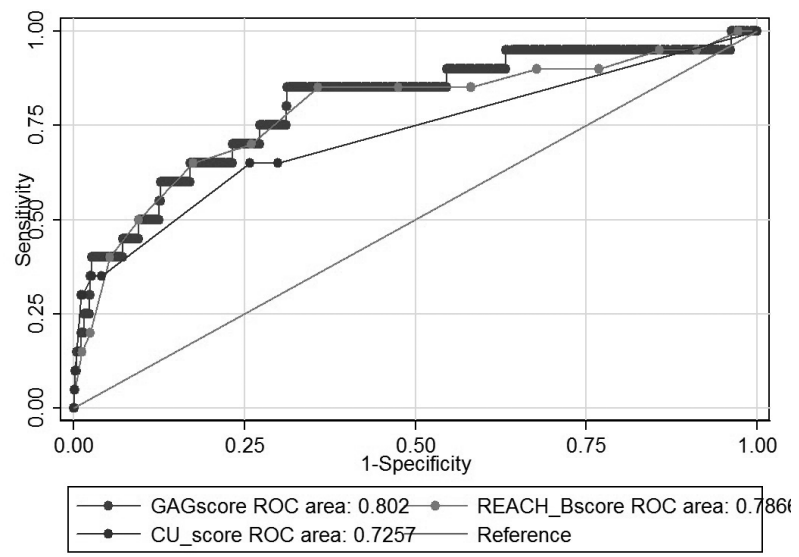

Abstract IDDF2019-ABS-0051 Figure 1 Comparison of the receiver operating characteristic curves of the risk score models 